\title{
Depiction and Convention
}

\begin{abstract}
By defining both depictive and linguistic representation as kinds of symbol system, Nelson Goodman attempts to undermine the platitude that, whereas linguistic representation is mediated by convention, depiction is mediated by resemblance. I argue that Goodman is right to draw a strong analogy between the two kinds of representation, but wrong to draw the counterintuitive conclusion that depiction is not mediated by resemblance.
\end{abstract}

It is a platitude that whereas words are connected to what they represent merely by arbitrary conventions, pictures are connected to what they represent by resemblance. My portrait and my name, for example, are importantly different because whereas my portrait and I are connected by my portrait's resemblance to me, there is only an arbitrary connection between my name and me. In this essay I defend this platitude from an alternative picture proposed by Nelson Goodman, which suggests that depictive and descriptive representation are equally arbitrary.

Goodman attempts to undermine the platitude that depiction is mediated by resemblance by defining both depiction and description as kinds of symbol system. From the claim that depiction is a kind of symbol system, Goodman draws the conclusion that "Almost any picture may represent almost anything; that is, given picture and object there is usually a system of representation, a plan of correlation, under which the picture represents that object." (Goodman, 1968, 38). Because they are both kinds of symbol system, Goodman argues, it follows that depiction and description are equally arbitrary.

Goodman's position is interesting because he draws an extremely close analogy between depictive and descriptive representation. The moral that can be drawn from my 
discussion of his views is that it is possible to uphold such a strong analogy between the two kinds of representation, while still maintaining the platitude that whereas descriptive representation is mediated by convention, depictive representation is mediated by resemblance. So Goodman's insights about the relationship between depiction and description can be accepted, but without drawing the counterintuitive consequences that he attempts to draw.

Section Two explains the argument which suggests to Goodman that depiction being a kind of symbol system is incompatible with depiction being mediated by resemblance. Section Three parodies that argument using an analogous argument which purports to show that linguistic representation is not conventional. Sections Four and Five develop the analogy between depiction and description by adapting David Lewis' analysis of the role of convention in linguistic representation into an analogous analysis of the role of resemblance in depictive symbol systems. Section Six uses that analysis to argue that Goodman is wrong to define depiction as a kind of symbol system.

One clarification. Depiction is a kind of representation, common to figurative painting and sculpture, photographs, maps, sketches and the like. So although abstract paintings and figurative paintings, for example, have much in common, abstract paintings are not counterexamples to the thesis that depiction is mediated by resemblance because they intuitively don't belong to the same kind of representation as figurative paintings. Abstract and figurative paintings are similar because they are all flat surfaces marked with paint, not because they all represent in the same way. ${ }^{1}$

A symbol system, according to Goodman's definition, is a set of characters correlated with a set of extensions $(1968,143)$. In the symbol system of Arabic numerals, for example, the characters are the numerals ' 1 ', '2', ' 3 ', '4'... and the extensions are the

\footnotetext{
${ }^{1}$ This is a point agreed upon even by those who, like Goodman, argue that resemblance is not the distinguishing feature of depiction. See, for example, Lopes (1996, 5-6).
} 
numbers $1,2,3,4, \ldots$ and so on. The symbol system correlates ' 1 ' with 1 , '2' with 2, '3' with 3, '4' with 4, and so on. According to Goodman, alphabets, languages, notations, musical scores and various methods of depiction are all distinct symbol systems. ${ }^{2}$

By a correlation between a set of characters and a set of extensions, Goodman simply means a function from the characters to the extensions. A function is a mapping between two sets: it delivers a member of the second set for every member of the first set. The function of doubling, for example, takes every member of the set of natural numbers to another member of that set: it takes 1 to 2, 2 to 4, 3 to 6, 4 to 8, and so on. Every mapping between two sets, no matter how arbitrary, is a function.

The advantage of defining symbol systems as functions from characters to extensions is that any symbol system can be fully specified by such a function. The symbol system of traffic lights, for example, is fully specified by the function that takes green to go, orange to slow and red to stop. Similarly the symbol system of Arabic numerals is fully specified by the function that takes ' 1 ' to 1, ' 2 ' to 2 and each other numeral to the number it represents.

Depiction, according to Goodman, is a kind of symbol system. The Mona Lisa, for example, is supposed by Goodman to represent Lisa because the Mona Lisa belongs to a symbol system in which the Mona Lisa is a member of the set of characters and Lisa is a member of the set of extensions and whose function maps the Mona Lisa to Lisa. The Mona Lisa depicts as well as represents Lisa because the symbol system involved is a depictive one.

Since a symbol system is simply a function from characters to extensions, and a function is any arbitrary mapping between two sets, any picture may represent anything in some symbol system or another. There is a function from portraits to people, for example, that takes the Mona Lisa to Socrates, and so it follows that there is a symbol system in which

\footnotetext{
${ }^{2}$ Goodman's claim that depiction is a kind of symbol system is also adopted by Bach (1970), Kulvicki (2003; 2006), Lopes (1996, 59) and Scholz (2000).
} 
the Mona Lisa represents Socrates. Just as words represent other things in other languages, pictures depict other things in other symbol systems: so what a picture represents appears to depend not on what it resembles but on its extension in a symbol system.

So if depiction is a kind of symbol system, then any depiction may represent anything and depictive representation appears just as arbitrary as linguistic representation. As Goodman puts it "Descriptions are distinguished from depictions not through being more arbitrary... and words are more conventional than pictures only if conventionality is construed in terms of differentiation rather than of artificiality.... what describes in some systems may depict in others. Resemblance disappears as a criterion of representation [depiction] ..." (Goodman, 1968, 230-1).

Even if it follows that depiction is arbitrary, it does not obviously follow that resemblance is not the feature which distinguishes depictive symbol systems from others, so that the initial platitude might be at least partially correct. According to this suggestion, a symbol system is depictive if and only if every character in that symbol system resembles its extension. So, for example, the symbol system of maps might be supposed to be depictive because all maps resemble the terrain they represent.

But the following example shows that as it stands even this weaker version of the platitude cannot be right. Often, letters of the alphabet are used to represent themselves, so that ' $a$ ' represents ' $a$ ', 'b' represents 'b', 'c' represents 'c', and so on. Since resemblance is reflexive, every letter in this symbol system resembles and represents itself, but it is intuitively not the case that every letter in the symbol system depicts itself, or that the symbol system described is depictive.

The reason is that the resemblance of the letters to themselves is incidental to their representation of themselves: if, for example, capital letters were used to represent lower case letters, so that the letters failed to resemble what they represent, the method of representation would not be substantially altered. So if Goodman is right that depiction is 
a kind of symbol system, it appears to follow that depictive representation is neither distinguished by resemblance nor less arbitrary than linguistic representation. In the following sections, I will use an analogy with convention to argue that neither conclusion follows and then use that analogy to develop an analysis of the role of resemblance in depictive symbol systems.

III

In the last section I gave an argument purporting to show that depictive representation is arbitrary and has no role for resemblance. In this section I will parody the argument that depiction is arbitrary and that there is no role for resemblance in depictive representation with an analogous argument that purports to show that there is no role for convention in linguistic representation. In the following sections I will exploit this analogy further to give a positive account of the role of resemblance in depictive symbol systems, before arguing that depiction is not a kind of symbol system.

Just as a symbol system is a function from characters to extensions, it is plausible that a language is a function from sentences to truth-conditions. ${ }^{3}$ So, for example, the meaning of 'Snow is white' in English is snow is white, according to the definition, because English is a function from sentences to truth-conditions that takes the sentence 'Snow is white' to the condition of snow being white. Similarly, 'Grass is green' means grass is green in English because 'Grass is green' is taken by the function to the truth-condition of Grass being green.

The main advantage of defining languages as functions from sentences to truthconditions, as for defining symbol systems as functions from characters to extensions, is that every language can be fully specified using such a function. English, for example, is fully specified by the function that takes 'Snow is white' to the state of affairs of snow being white, 'Grass is green' to the state of affairs of Grass being Green, and so on. (Putting aside issues concerning mood and indexicals, which aren't relevant here).

\footnotetext{
${ }^{3}$ See, for example, Lewis (1975).
} 
Since truth-conditions are the extensions of sentences and sentences are the characters of languages, this definition of languages is just a special case of Goodman's definition of symbol systems. This brings out another advantage of Goodman's position: the definition of symbol systems is a natural generalisation of the definition of languages, and the definition of depiction as a kind of symbol system is a natural analogue of the definition of languages as functions from sentences to truth-conditions. ${ }^{4}$

Just as the claim that depiction is a kind of symbol system appears to leave no role for resemblance in depictive representation, the definition of languages as functions from sentences to truth-conditions at first appears to leave no role for convention in linguistic representation. The problem is that a function from sentences to truth-conditions necessarily takes the sentences it does to the truth-conditions it does: the function from English sentences to the states of affairs they represent, for example, necessarily takes the sentence 'Snow is white' to the state of affairs of snow being white.

In general, if sentences necessarily mean what they do in a language, then it appears that no convention links sentences to what they mean, and so convention has no role in fixing their meaning. If 'Snow is white', for example, necessarily means in English that snow is white, then no convention is needed to link the two. If languages are functions from sentences to truth-conditions, then having a convention that 'Snow is white' means snow is white in English is like having a convention to the effect that falling objects must accelerate at approximately ten metres per second per second.

But to conclude on these grounds that convention has no role in linguistic representation would be obviously incorrect. This is because, while it is a matter of necessity rather than convention that 'Snow is white' in English means that snow is white, it is contingent that English, or the language given by the function from English sentences to their truthconditions, is the language of this continent, and this is a fact which is clearly contingent and is therefore plausibly governed by convention.

\footnotetext{
${ }^{4}$ Lopes $(1996,59)$ points out the connection between the definitions of languages and symbol systems.
} 
In general, while the meaning of a sentence in a language is always a matter of necessity, it is always a contingent matter whether a language or a function from sentences to truthconditions is used or spoken by a population. So although it is a matter of necessity, for example, that 'Snow is white' means snow is white in English, it is nonetheless an arbitrary matter that we speak a language in which 'Snow is white' means snow is white rather than a different language in which it means that grass is green.

The role of convention in linguistic representation is not to determine the meaning of a sentence in a language, but to determine whether or not a language or a function from sentences to truth-conditions is used or spoken by a population. So, for example, English is the language of this continent, rather than some other language which would serve equally well, due to the fact that there is a convention of speaking English on this continent. Language is conventional because convention determines which language is used by a population. ${ }^{5}$

If the platitude that resemblance plays a similar role in depictive representation as convention plays in linguistic representation is right, then this suggests that the role of resemblance in depictive representation might be in determining which symbol system a population uses. In the next two sections I will follow up this suggestion by introducing an analysis of depictive symbol systems analogous to Lewis' analysis of conventional language, which shows that Goodman is wrong to infer from depiction being a kind of symbol system that depiction is not mediated by resemblance.

A convention is a rationally self-perpetuating regularity in behaviour. Driving on the left, for example, is a convention in Australia because there is a regularity of driving on the left and because the existence of this regularity provides Australian drivers with a rational reason for continuing to drive on the left. Driving on the right is a convention in Europe

\footnotetext{
${ }^{5}$ These points are from Lewis $(1969 ; 1975)$.
} 
because there is a regularity of driving on the right in Europe, and the existence of this regularity gives European drivers a reason for continuing to drive on the right. ${ }^{6}$

For a regularity of driving on the left to be a convention it must be, firstly, that drivers are aware that there is a regularity of driving on the left and, secondly, that drivers have a reason to drive on the left on condition that the others do. This is because if either of these conditions failed then the regularity of driving on the left would not be rationally self-perpetuating: drivers would not continue driving on the left in the first case because they would not know that it was better to do so and in the second case because others driving on the left would not give them any reason to do so.

These points suggest the following initial formulation of Lewis' $(1969,58)$ analysis of convention, which is that a regularity in the behaviour of a population is a convention if and only if:

(1) everyone conforms to that regularity

(2) everyone expects everyone else to conform to that regularity

(3) everyone has reason to conform on condition that all others conform.

So, for example, driving on the left is a convention in Australia because everybody drives on the left, everybody expects everybody else to drive on the left, and everybody has reason to drive on the left if everybody else drives on the left.

Three amendments. First, imagine that everyone drives on the left because they expect others to and because everybody has reason to drive on the same side as others. However, nobody believes that others drive on the left for these reasons: rather everyone believes that others drive on the left merely out of habit or because driving on the left is more scenic. The regularity of driving on the left in this population is not a convention for avoiding collision, since members of the population would continue driving on the left even though they believe others may not care about collision (Lewis, 1969, 59).

\footnotetext{
${ }^{6}$ This section summarizes Lewis (1969). Bennett (1971), Abell (2005) and Novitz (1977) discuss Lewis' analysis of convention in relation to depiction.
} 
To avoid this case it is necessary to build into the analysis that all the members of the population are aware that all the conditions of the analysis are fulfilled. A regularity in the behaviour of group is a convention, according to the amended analysis if and only if:

(1) everyone conforms to that regularity

(2) everyone has a reason to conform on condition that all others conform

(3) everyone believes (1)-(3).

So, for example, driving on the left is a convention in Australia because everybody drives on the left, everybody prefers to drive on the left given that everybody else drives on the left and because everybody believes that everybody else conforms to the regularity for these reasons.

Secondly, the condition that everyone has reason to conform on condition that others do is supposed to capture the cooperativeness of convention, since in conventional behaviour everyone has reason to do conform to the same regularity because of their common interests. The convention of driving on the left in Australia, for example, arises because everybody has a common interest in driving on the same side, and this common interest gives them a reason to conform to the regularity of driving on the left if others do. All conventions are similarly based on such common interests (Lewis, 1969, 69).

But the conditions of the analysis may be met even when common interests are absent. Imagine a work place in which everyone aims to impress their employer by outperforming their peers. A regularity of hard work obtains in this work place, everybody has reason to conform to the regularity of hard work on condition that others do, and everybody is aware of this. Nevertheless, hard work is not a convention of the workplace, because the workers do not have a common interest in everybody working hard.

To address this problem it is necessary to build into the analysis a condition stipulating that everybody has a common interest in general, rather than merely partial, conformity to the regularity (Lewis, 1975, 165). So a regularity in the behaviour of a group is a convention, according to the amended analysis, if and only if:

(1) everyone conforms to that regularity 
(2) everyone has a reason to conform on condition that all others conform

(3) everyone prefers everyone to conform to that regularity, on condition that most do

(4) everyone believes (1)-(4).

So driving on the left, for example, is a convention in Australia because everyone prefers everyone to drive on the left on condition that most do, since everyone driving on the left will tend to reduce accidents on condition that most people drive on the left.

Thirdly, the condition that everybody has reason to conform because others do is supposed to capture the arbitrariness of convention, since everyone is supposed to conform to the regularity for no other reasons than that others do. The convention of driving on the left, for example, is supposed to be arbitrary because nobody has much reason to drive on the left except the fact that others drive on the left. The regularity of driving on the left is no better than the regularity of driving on the right, which everyone would happily adopt were it adopted by others.

But the condition does not guarantee arbitrariness in all cases. Suppose, for example, that there is only one café in our town and that it is a regularity in our behaviour to meet at that café to drink coffee. We only like to drink coffee together, so that I have reason to go to the café only if you go to the café. Nevertheless, because there is no alternative place for us to drink coffee our regularity of meeting at the café is not chosen arbitrarily but of necessity, and because it is not chosen arbitrarily, it is not correctly classified as a convention.

To address this problem the analysis has to be amended to ensure that an alternative regularity is available. So a regularity in behaviour of a group is a convention, according to the final amended analysis, if and only if:

(1) everyone conforms to that regularity

(2) everyone has reason to conform on condition that all others conform

(3) there is an alternative regularity which everyone would have had reason to conform to if others had conformed to it 
(4) everyone prefers everyone to conform to that regularity, on condition that most do

(5) everyone believes (1)-(5) (Lewis, 1969, 76; 1975, 165).

So, for example, driving on the left would not be a convention if it weren't for the existence of the option of driving on the right, which everyone else would have reason to do if others did.

As I argued in the last section, language is conventional in the sense that conventions determine which language or function from sentences to truth-conditions are used by people in speech and communication. Convention, for example, determines that English, the function from English sentences to their truth-conditions, rather than Liarese, a function from English sentences to the contrary truth-conditions, is a language spoken on the continent of Australia.

Since conventions apply primarily to regularities of behaviour the analysis of convention may not apply directly to functions from sentences to truth-conditions, but only to a regularity in behaviour concerning those functions. According to Lewis, the relevant regularity is truthfulness in the language, which consists in trying to sometimes utter sentences of the language when and only when the truth-conditions or states of affairs they represent in that language obtain. (Due to mood and other complications a slightly different regularity is required, but truthfulness serves for current purposes.)

So being truthful in English, for example, consists in sometimes uttering sentences such as 'Snow is white' if snow is white and abstaining from uttering sentences such as 'The sky is green' if the sky is not green, whereas being truthful in Liarese, the function from English sentences to the contraries of their truth-conditions in English, consists in abstaining from uttering sentences such as 'Snow is white' when snow is white and uttering sentence such as 'The sky is green' when the sky is not green.

Lewis $(1969,177)$ suggests that a group has a convention of using a language if and only if they have a convention of truthfulness in that language or, putting this together with the 
full analysis of convention that a group has a convention of using a language if and only if there is a regularity in the group such that:

(1) everyone is truthful in that language

(2) everyone has reason to be truthful in that language on condition that all others are truthful in that language

(3) there is an alternative regularity of truthfulness in another language which everyone would have reason to conform to if others conformed to it

(4) everyone prefers everyone to conform to a regularity of truthfulness in that language on condition that most do

(5) everyone believes (1)-(5).

English, for example, is a language spoken by English speakers since English speakers are truthful in English, English speakers expect all other English speakers to be truthful in English, and all English speakers prefers to be truthful in English given that everybody else is.

\section{$\mathrm{V}$}

It is a platitude that, whereas words are connected to what they represent merely by arbitrary conventions, depictions are connected to what they represent by resemblance. That suggests that the role of resemblance in depictive representation is analogous to the role of convention in linguistic representation, so that substituting symbol systems for languages in Lewis' analysis of conventional language use and adding that which symbol systems are used is not arbitrary but depends on the resemblance between their characters and extensions should provide an analysis of depictive symbol systems.

Arbitrariness is ensured in the analysis of convention by the second condition, which ensures that the population conforms to the regularity for no other reason than that other members of the population conform to it, and by the third condition, which ensures that there are other regularities which all members would have preferred to conform to had others done so (Lewis, 1969, 70). To adapt Lewis' analysis of the conventions governing 
linguistic representation in order to provide an analysis of depictive symbol systems, these are the conditions that ought to be altered.

The second condition of Lewis' analysis captures the arbitrariness of linguistic conventions by specifying that which language the members of a population have reason to use depends on which language other members of that population choose to use, rather than any feature of the language independent of the choices of others. To provide an analysis of depictive symbol systems, this condition should be altered to include resemblance, because the resemblance of a symbol system's characters to their extensions in that symbol system provides an additional reason for its use.

The third condition of Lewis' analysis further ensures the arbitrariness of linguistic conventions by specifying that there must be an alternative to the regularity members of the population actually conform to which would serve their purposes just as well. Since the relationship between depictions and what they represent is non-arbitrary, this condition becomes unnecessary and should be dropped: although there may be alternative regularities which would serve just as well, there need not be such alternatives in order for the use of a symbol system to count as depictive.

This suggests that a symbol system is depictive if and only if there is a regularity of truthfulness in that symbol system such that:

(1) everyone is truthful in that symbol system

(2) everyone has reason to be truthful in that symbol system, since its characters resemble their extensions

(3) everyone prefers everyone to conform to a regularity of truthfulness in that language on condition that most do

(4) everyone believes (1)-(4).

So, for example, the symbol system of maps is depictive because everybody uses them, everybody expects everybody to use them, and everybody has reason to do this because maps resemble what they represent, rather than simply because everybody else uses them. 
One clarification. The definition of truthfulness in a language cannot be straightforwardly applied to symbol systems, since symbol systems are functions from characters to extensions rather than truth-conditions and extensions need not be true or false. One cannot be truthful in the symbol system of Arabic numerals, for example, since the extensions of the numerals in the system are numbers, which exist or not rather than obtaining or being true or false.

The solution lies in observing that depiction is of states of affairs as well as objects. The Mona Lisa, for example, does not merely depict Lisa but also the state of affairs of Lisa smiling. Similarly, depictions resemble states of affairs as well as objects: the Mona Lisa does not merely resemble Lisa (the object), but also resembles Lisa smiling (the state of affairs). So the difficulty can be overcome by applying the analysis first towards the depiction of states of affairs and then stipulating that an object is depicted if and only if it occurs in a depicted state of affairs.

The analysis of depictive symbol systems just given shows that neither of the conclusions Goodman draws from the claim that depiction is a kind of symbol system follows. It appeared to follow, for example, that depiction could not be characterized in terms of resemblance because there was no non-incidental role for resemblance in depictive representation. But the analysis shows that there is a non-incidental role for resemblance, since resemblance between characters and extensions of a symbol system determines which symbol systems are adopted for use.

The symbol system of using letters to represent themselves, for example, is not counted as depictive because although all the characters in that symbol system resemble their extensions, it is not for this reason that this symbol system is preferred over others as a method of representing the letters. If, on the other hand, the symbol system of using letters to represent themselves was preferred due to their resemblance to themselves, it seems it would count as a depictive symbol system. So this analysis, unlike its predecessor, accommodates this example. 
Furthermore, the analysis shows that it does not follow from depiction being a kind of symbol system that the relationship between depictions and what they represent is merely arbitrary. While it is true that there are always other symbol systems in which the same pictures would have different extensions, which symbol system is selected for use in communication is not arbitrary but depends on the resemblance between the characters and extensions of that system just as, while it is true that sentences have their meaning in English necessarily, linguistic meaning is not a matter of necessity since which language is spoken depends on arbitrary conventions.

So far I have argued that the thesis that depiction is a kind of symbol system does not entail that depiction is not mediated by resemblance, but I have bracketed the question of whether or not depiction is a kind of symbol system, on which the analogy Goodman draws between depiction and language rests. In this section, I will argue that Goodman is incorrect to define depiction as a kind of symbol system, on the grounds that not all depictions belong to depictive symbol systems. But, I will argue, rather than undermining the analogy between depiction and language, this result in fact supports it.

Because it's trivially true that there is a function which maps every depiction to what it represents, Goodman's claim that depiction is a kind of symbol system is difficult to adjudicate. The analysis of depictive symbol systems just given makes adjudicating this claim possible, because it is not trivial whether every depiction meets the four conditions required to belong to a depictive symbol system. I will argue that the analysis shows that some but not all depictions belong to depictive symbol systems, so that depiction cannot be defined as a kind of symbol system.

Maps, for example, belong to depictive symbol systems, since amongst surveyors there is a regularity of truthfulness in the symbol system of maps, the fact that maps resemble their state of affairs in that symbol system give surveyors a reason to conform to that regularity, surveyors prefer each other to conform to that regularity on condition that 
most do and all surveyors are aware of all these facts. The resemblance between maps and what they represent is a reason for surveyors to conform to the regularity, because all surveyors desire to conform to a system in which the symbols are readily interpreted by other surveyors.

But not all depictions belong to depictive symbol systems. Suppose, for example, that Philomela's only motive is to exact revenge on Tereus for cutting out her tongue. In order to do so, she weaves a tapestry depicting his crime. Trivially, there are many functions from characters to extensions that takes Philomela's tapestry to Tereus' crimes. I will argue that since none of those functions need meet the four conditions of being a depictive symbol system, Philomela's tapestry is a depiction that does not belong to a depictive symbol system. And since not all depictions belong to symbol systems, depiction is not a kind of symbol system.

The first condition of the analysis requires a regularity of truthfulness in a symbol system. Philomela's tapestry meets this condition; there is a regularity of truthfulness, for example, in the function that takes Philomela's tapestry to Tereus' crimes and the Bayeux tapestry to the Battle of Hastings. But if, for example, the Bayeux tapestry and every other depiction apart from Philomela's tapestry had not existed, Philomela's tapestry would have depicted Tereus' crimes without belonging to any regularity of truthfulness. So it is not the case that all depictions must meet the first condition of belonging to a depictive symbol system.

The second condition requires that the resemblance of characters to extensions in a symbol system gives everyone reason to be truthful in that symbol system. Because her only motive is revenge on Tereus, Philomela has no general reason to conform to a regularity of truthfulness. She has no reason, for example, to perpetrate the Bayeux tapestry when the Battle of Hastings occurs, so she has no general reason for being truthful in the symbol system which takes her tapestry to Tereus' crimes and the Bayeux tapestry to the Battle of Hastings. So Philomela's tapestry is a depiction which fails to meet the second condition of belonging to a depictive symbol system. 
The third condition requires that everybody prefers everybody to conform to a regularity of truthfulness in the symbol system if most do. Philomela's tapestry doesn't meet this condition because if Philomela's only motive is revenge on Tereus, then she is indifferent as to whether anybody else conforms to a regularity of truthfulness in any symbol system, including in the symbol systems that takes her tapestry to Tereus' crimes and the Bayeux tapestry to the Battle of Hastings. But despite not meeting the third condition of belonging to a symbol system, Philomela's tapestry is a depiction, so the third condition of belonging to a symbol system is not met by all depictions.

Since it need not meet the first three conditions, Philomela's tapestry need not meet the fourth condition either. The fourth condition requires that everyone in a population believes that the first three obtain. So Philomela's tapestry could remain a depiction without meeting the fourth condition, if anybody in the population falsely believed that the tapestry did not belong to any regularity of truthfulness, because there were no other depictions, or if anybody in the population rightly believed that Philomela had no general reason to conform to a regularity of truthfulness or that she had no preference to conform to such a regularity if others did.

So some depictions, such as maps or architectural plans, belong to depictive symbol systems whereas others, such as Philomela's tapestry or improvised sketches, do not. The analysis of depictive symbol systems as those which are used because their characters resemble their extensions shows that Goodman is wrong to define depiction as a kind of symbol system. In the next section, I will reply to the objection that my argument proves too much because it also disproves Lewis' analysis of conventional language and in the final section I will argue that my conclusion that depiction is not a kind of symbol system still supports a close analogy between depiction and language. 
The arguments just given that depictions need not belong to depictive symbol systems might be taken to prove too much, on the grounds that there is a parallel problem for Lewis' analysis of conventional language. The problem is that non-literal and loose uses of language suggest that people do not conform to regularities of truthfulness in the languages that they actually speak. In cases of hyperbole, metaphor and irony, for example, it is standard to say things which one knows to be false. The prevalence of such examples suggests that there is in fact no regularity of truthfulness in English.

Lewis' response to the problem is to agree that there is no regularity of truthfulness in literal English, but argue that there is a regularity of truthfulness in a more complex language, which consists of a function that takes English sentences to their non-literal rather than their literal meanings. This more complex language would take an ironic utterance of the sentence 'John is a fine friend' to the truth-condition of John being a false friend, an utterance of 'Juliet is the sun' to the truth-condition of Juliet being pretty, and so on. Literal English is then taken as a simplified version of this more complex language.

The arguments I gave to show that depiction is not a kind of symbol system also show that Lewis' response to this problem is incorrect. While Lewis is right that people do conform to a regularity of truthfulness in the more complicated language, he is incorrect that there is any convention of conforming to that regularity. The reason is that although the regularity of truthfulness in the more complex language trivially meets the first and fourth condition of the analysis of convention, it need not meet the other three conditions.

There might, for example, be a population of speakers who engage heavily in metaphor, irony, hyperbole, puns and other non-literal use. Such a group of speakers would trivially conform to a regularity of truthfulness in a language more complex than their literal one. But they may all fail to meet the second condition of the analysis, because it might be that they would prefer to use non-literal language even if other members of the population began a strict regiment of speaking literally. 
The population in question would not regard such a change in other members as a matter of indifference with the thought that as long as effective communication were not impaired it does not matter whether people speak literally or not, just as speaking in an alternative literal language such as French or German would not be any worse than speaking English. Rather all consider that the result of speaking literally would be boring and tedious and all feel that were this to happen it would be up to them to keep things lively by talking as non-literally as possible.

The third condition would not obtain in such a population either, because although speaking in literal English is an alternative to speaking in non-literal English, it is not the case that everybody would have reason to speak in literal English if others did, since it is not the case that everybody would prefer to speak literally if others did. Speaking literal English is an alternative to speaking non-literal English, but it isn't an alternative which is just as good as speaking non-literal English, because non-literal English has more scope for entertainment and expression.

The fifth condition, which requires that all members of the population be aware that the conditions of the analysis obtain, need not be met either. There might be a population, for example, in which everyone speaks non-literally all the time. Everyone in the population does so and have reason to do so merely because others do: the population is a shy and nervous bunch, who don't want to stand out from their peers. Secretly, everyone in the population would be equally happy to speak literally but, not knowing this about the others, they fail to meet the final clause of the analysis.

So it follows, contrary to Lewis and for the same reasons that not all depictions depict by belonging to depictive symbol systems, that non-literal utterances don't have their meanings in virtue of conventions of truthfulness in a language more complicated than literal English. Nevertheless, it does not follow that the argument that not all depictions belong to depictive symbol systems prove too much, because although the argument also 
proves that there is no convention of truthfulness in a non-literal language, this extra conclusion is correct. ${ }^{7}$

This means that Lewis cannot defend his analysis of when a language is conventionally used by a population by appeal to conventions of truthfulness in non-literal languages and that another solution is required to the problem posed by the fact that due to metaphor, irony, hyperbole and other non-literal language there is no regularity of truthfulness in literal English. I think the solution is to maintain that there is a conventional regularity of speaking literal, rather than non-literal English, but that truthfulness has to be redefined to capture the relevant regularity in order to accommodate metaphor, irony and nonliteral talk.

The correct response to the problem is that truthfulness in a language should not be defined as uttering sentences in that language when one intends one's audiences to believe or bring about that the truth-conditions obtain, since this definition has the consequence that there is no regularity of truthfulness in English. Instead, truthfulness should be defined as uttering sentences in a language when the audience is intended to infer from the fact that a sentence has the mood and truth-condition that it does that the utterance is intended to induce an effect in them, but not necessarily to believe or bring about that that truth-condition obtains.

So, for example, literal utterances of 'it's raining' conform to a regularity of truthfulness in English because audiences of utterances of 'it's raining' are intended to infer from the fact that the sentence's truth-condition in English is that it's raining that they are intended to believe it's raining. Metaphorical utterances of 'Juliet is the sun' also conform to a regularity of truthfulness in English, because audiences are intended to infer from the fact that the sentence's truth-condition in English is Juliet being the sun that they are intended to believe something else, such as that Juliet is beautiful.

\footnotetext{
${ }^{7}$ Sperber and Wilson (2002) argue for this point in detail. They propose a convention of relevance to replace Lewis' convention of truthfulness.
} 
So. The analysis of depictive symbol systems shows that Goodman is wrong on two counts: depiction is not a kind of symbol system and, even if it were, it does not follow from depiction being a kind of symbol system that it is mediated by resemblance. But Goodman is also partially right: there is a kind of symbol system which is distinctively depictive and many depictions, such as maps or architectural plans, belong to those symbol systems. Furthermore, the analogy Goodman draws between depiction and language is reflected in the analogy between the analyses of convention and depictive symbol systems.

The conclusion that depiction is not a kind of symbol system might seem to undermine this important analogy, since the analysis of conventional language appears to encompass everything it is expected to whereas the analysis of depictive symbol systems appears not to. While maps and other members of depictive symbol systems, for example, have their linguistic analogues, it may seem that Philomela's tapestry, improvised sketches and other less systematic depictions lack linguistic cousins, so that the analogy between depictive and descriptive representation is not as close as Goodman and I both maintain.

But this conclusion would be premature. Just as some depictions fall under the analysis of depictive symbol systems whereas others do not, some spoken utterances belong to conventional language whereas others fall outside it. If somebody calls out loudly in alarm, for example, then, whilst there call trivially belongs to some language or another, whether it falls under the analysis of conventional language use will depend on the preferences, reasons and beliefs of the speaker and their population. Such improvised utterances are the analogues of depictions which fall outside the analysis of depictive symbol systems.

So the extremely close analogy between depictive and descriptive modes of representation Goodman drew by defining depiction as a kind of symbol system turns out to be correct. In his words: "The often stressed distinction between iconic and other signs 
becomes transient and trivial; thus does heresy breed iconoclasm.” $(1968,231)$. But I have also argued that the counterintuitive consequences Goodman draws from this analogy do not follow: in particular, the platitude that depictions are connected to what they represent via resemblance whereas words are connected to what they represent only arbitrarily is preserved. 


\section{References}

Abell, Catharine (2005b), “Against Depictive Conventionalism”, The American Philosophical Quarterly, 42(3): 185-197.

Bach, Kent (1970), "Part of What a Picture Is", British Journal of Aesthetics, 10: 119-137.

Bennett, John (1971), “Depiction and Convention?”, The Monist 58: 255-68.

Goodman, Nelson (1968), Languages of Art: An Approach to a Theory of Symbols (Indianapolis and New York: The Bobbs-Merrill Company, Inc.).

Grice, H. P. (1989), Studies in the Way of Words (Cambridge, Mass.: Harvard University Press).

Kulvicki, John (2003), "Image Structure”, The Journal of Aesthetics and Art Criticism, 61(4): 323-39.

Kulvicki, John (2006), On Images: Their structure and content (Oxford: Oxford University Press).

Lewis, David (1969), Convention (Cambridge, Mass.: Harvard University Press).

Lewis, David (1975), "Languages and Language", Minnesota Studies in the Philosophy of Science VII: 3-35. Reprinted in Lewis (1983).

Lewis, David (1983), Philosophical Papers I (Oxford: Oxford University Press).

Lopes, Dominic (1996), Understanding Pictures (Oxford: Clarendon Press). 
Novitz, David (1977), Pictures and their Use in Communication (The Hague: Martinus Nijhoff).

Scholz, Oliver (2000), “A Solid Sense of Syntax”, Erkenntnis, 52: 199-212.

Sperber, Dan and Deirdre Wilson (2002), “Truthfulness and Relevance”, Mind 111(443): 583-632. 